\section{(1) \\ CrossMark}

\title{
Systematic review of drug effects in humans and models with surfactant-processing disease
}

\author{
Dymph Klay ${ }^{1}$, Thijs W. Hoffman ${ }^{1}$, Ankie M. Harmsze², Jan C. Grutters ${ }^{1,3}$ and \\ Coline H.M. van Moorsel ${ }^{1,3}$
}

Affiliations: ${ }^{1}$ Interstitial Lung Disease Center of Excellence, Dept of Pulmonology, St Antonius Hospital, Nieuwegein, The Netherlands. ${ }^{2}$ Dept of Clinical Pharmacy, St Antonius Hospital, Nieuwegein, The Netherlands. ${ }^{3}$ Division of Heart and Lung, University Medical Center Utrecht, Utrecht, The Netherlands.

Correspondence: Coline H.M. van Moorsel, Interstitial Lung Disease Center of Excellence, St Antonius Hospital, Koekoekslaan 1, Nieuwegein, 3435CM, The Netherlands. E-mail: c.van.moorseldantoniusziekenhuis.nl

@ERSpublications

Drug effects in disease models of surfactant-processing disease are highly dependent on mutation http://ow.ly/ZYZH30k3RkK

Cite this article as: Klay D, Hoffman TW, Harmsze AM, et al. Systematic review of drug effects in humans and models with surfactant-processing disease. Eur Respir Rev 2018; 27: 170135 [https://doi.org/10.1183/ 16000617.0135-2017].

ABSTRACT Fibrotic interstitial pneumonias are a group of rare diseases characterised by distortion of lung interstitium. Patients with mutations in surfactant-processing genes, such as surfactant protein $\mathrm{C}$ (SFTPC), surfactant protein A1 and A2 (SFTPA1 and A2), ATP binding cassette A3 (ABCA3) and Hermansky-Pudlak syndrome (HPS1, 2 and 4), develop progressive pulmonary fibrosis, often culminating in fatal respiratory insufficiency. Although many mutations have been described, little is known about the optimal treatment strategy for fibrotic interstitial pneumonia patients with surfactant-processing mutations.

We performed a systematic literature review of studies that described a drug effect in patients, cell or mouse models with a surfactant-processing mutation. In total, 73 articles were selected, consisting of 55 interstitial lung disease case reports/series, two clinical trials and 16 cell or mouse studies. Clinical effect parameters included lung function, radiological characteristics and clinical symptoms, while experimental outcome parameters included chemokine/cytokine expression, surfactant trafficking, necrosis and apoptosis. SP600125, a c-jun N-terminal kinase (JNK) inhibitor, hydroxychloroquine and 4-phenylbutyric acid were most frequently studied in disease models and lead to variable outcomes, suggesting that outcome is mutation dependent.

This systematic review summarises effect parameters for future studies on surfactant-processing disorders in disease models and provides directions for future trials in affected patients.

\section{Introduction}

Idiopathic interstitial pneumonias (IIPs) are a rare group of diseases characterised by distortion of lung interstitium. IIPs can be subclassified into fibrotic interstitial pneumonia (FIP), smoking-related interstitial pneumonia and acute/subacute interstitial pneumonia [1]. The aetiology of IIPs is unknown; however, affected patients commonly have a first-degree relative with pulmonary fibrosis, referred to as familial interstitial pneumonia. It has been suggested that up to $20 \%$ of FIP cases might be familial $[2,3]$. In FIP, two distinct groups of causal genetic mutations have been recognised; surfactant-processing and

\section{This article has supplementary material available from err.ersjournals.com}

Provenance: Submitted article, peer reviewed.

Received: Dec 182017 | Accepted after revision: April 122018

Copyright CERS 2018. ERR articles are open access and distributed under the terms of the Creative Commons Attribution Non-Commercial Licence 4.0. 
telomere maintenance gene mutations. To date, mutations in four surfactant-associated genes have been found to cause pulmonary fibrosis: surfactant protein $\mathrm{C}$ (SFTPC) $[4,5]$, surfactant protein A1 (SFTPA1) [6], surfactant protein A2 (SFTPA2) [7, 8] and ATP binding cassette transporter (ABCA3) [9, 10]. Furthermore, mutations in Hermansky-Pudlak syndrome 1 (HPS1) and 4 (HPS4) can cause pulmonary fibrosis in patients with Hermansky-Pudlak syndrome (HPS) with a lung phenotype equalling that of FIP $[11,12]$. In addition, it has been found that Hermansky-Pudlak syndrome 2 (HPS2, associated with mutations in gene $A P 3 B 1)$ can cause pulmonary fibrosis in children and only results in mild interstitial lung disease (ILD) in adults [13]. SFTPC, SFTPA1 and SFTPA2 encode surfactant proteins (SPs)-C, -A1 and $-\mathrm{A} 2$, respectively, that have various biophysical functions and protect alveoli against damage and infection [14, 15]. The genes ABCA3, HPS1, AP3B1 and HPS4 encode structural proteins of lamellar bodies, the characteristic organelles in alveolar type II cells (AEC2) that are crucial for surfactant processing [16-18]. In general, mutations in surfactant-processing genes seem to cause misfolding or inappropriate localisation of proproteins, which leads to accumulation of proprotein in the endoplasmic reticulum or in inappropriate cellular compartments or in degradation of the proprotein [19]. In turn, this results in altered cellular processes in AEC2, such as dysregulated proteostasis, altered surfactant lipid composition and activation of immune cells in SFTPC non-BRICHOS mutations [20, 21], endoplasmic reticulum stress in SFTPC BRICHOS mutations and SFTPA1 and 2 mutations [22-26] and impaired lipid transport, dysfunctional lysosome-related organelles, increased endoplasmic reticulum stress and apoptotic signalling in $A B C A 3$ and HPS mutations [17, 27-29]. In addition to the above disease-causing mutations, single nucleotide polymorphisms in the MUC5B and TOLLIP genes have been associated with predisposition to idiopathic pulmonary fibrosis (IPF), as well as survival in IPF patients [30-32]. Recent studies have identified various molecular phenotypes in IPF patients. These different molecular phenotypes correspond to variance in disease behaviour, and possibly to the response to different treatment regimens. Stratification of FIP patients based on genetic characteristics as well as cellular and molecular biomarkers could lead to personalised treatment strategies in the future [33, 34]. However, previous therapeutic trials have mostly not tested what genetic characteristics and biomarkers are associated with a good treatment response. Therefore, it is not known whether FIP patients with surfactant-processing mutations should receive the same therapy as other FIP patients. They might benefit from a different treatment strategy.

The aim of this systematic review is to provide an overview of studies that investigated drug effects in patients, cell or mouse models containing a mutation in surfactant-processing genes involved in pulmonary fibrosis. This review will focus on drug types, effect parameters and outcomes. This will provide a basis for future research efforts into treatment strategies for FIP patients with surfactant-processing mutations.

\section{Materials and methods \\ Data sources and literature searches}

A literature search in the electronic databases of Pubmed and Embase was performed with the help of a clinical librarian. We selected studies that contained, in the Medical Subject Headings (MeSH), keywords or text words, at least one search item from each of the following three groups: 1) ILD, with MeSH terms referring to ILD or lung cells; 2) surfactant-processing mutation, with $\mathrm{MeSH}$ terms referring to genes involved in adult FIP, pulmonary surfactant-associated protein, Hermansky-Pudlak syndrome or ATP-binding cassette transporter; 3) treatment, with MeSH terms referring to drug, treatment or therapy. The search was restricted to articles written in English and published before July 2, 2017. In the Embase search, conference abstracts were excluded. To maximise the inclusion of case reports/series, a second search was performed. This search included search items from groups 1 and 2, but not group 3 (treatment). This second search was restricted to the categories case report, clinical article, clinical study, clinical trial (phase I-IV), (classical) article, cohort analysis, comparative study, controlled study, controlled clinical trial, human, human tissue, major clinical study, retrospective study, evaluation study, letter, multicentre study, observational study, pragmatic clinical trial and randomised control trial. Duplicates within the search with two search items and the search with three search items were identified and removed using the reference management programme RefWorks (Ann Arbor, MI, USA). Duplicates between the two searches were removed manually. The complete search strategy is provided in the online supplementary material.

\section{Study selection}

Title and abstract of the retrieved articles were reviewed and articles were scored based on the following three criteria. Studies involving 1) ILD, mouse models or cells and lung disease; 2) surfactant-processing mutation involved in adult pulmonary fibrosis; and 3) original research articles. Abstracts that were scored for all three criteria were selected and the full-text versions were reviewed. Studies were excluded that reported no or only non-pharmacological drug effects, such as small interfering RNA/short hairpin RNA, 
gene overexpression, supplemental oxygen, bronchoalveolar lavages and lung transplantation. Case reports/ series were excluded in which HPS diagnosis was not based on genetic analysis or absence of dense granules in platelets assessed by electron microscopy. The references of the finally selected articles were screened for additional eligible studies.

\section{Classification of drug effect in case reports/series}

Drug effect in case reports or series of paediatric and adult cases was determined based on the information found in journal articles. Different terms, such as improvement, short-term improvement, stabilisation, short-term stabilisation, limited effect or no effect were used to express outcome of treatment. Sick-better, (some) improvement of lung function, clinical or respiratory symptoms, chest film or high-resolution computed tomography (HRCT) or just improvement after treatment was defined as improvement; transient effect or improvement and later a reduced effect of treatment or deterioration of disease was defined as short-term improvement; stable condition, stabilisation of lung function, clinical symptoms or sick-same was defined as stabilisation. Short-term stabilisation was used when this drug effect was only present for a short period of time. No effect after treatment, deterioration of lung function, clinical symptoms or HRCT was defined as no effect; and limited effect was used when little effect or minimal improvement followed by death was reported.

\section{Results}

\section{Search results}

In figure 1, the flow diagram of the search and study selection process is displayed. Two different searches, one with three groups of search terms (ILD/lung cells, surfactant-processing mutation and treatment) and one with two groups of search terms (ILD/lung cells and surfactant-processing mutation) were performed. The searches resulted in a total of 1878 unique articles. The full text was read of 239 articles, of which 73 were selected to be included in this review. Selected studies consisted of 16 studies performed in cell or mouse models, 55 case reports/series and two clinical trials. Mutations in SFTPC, HPS1 and ABCA3 were most frequently studied. The number of case reports/series, clinical trials and cell/mouse studies per mutation are displayed in table 1 .

\section{Drugs and effect parameters used in case reports/series and cell/mouse studies}

The studied drugs can be divided into immunosuppressive agents, antifibrotic agents, mitogen-activated protein kinase signalling pathway inhibitors, antibiotics, combination therapy, anti-apoptotic therapy and

FIGURE 1 Flowchart of the article selection process. HPS: HermanskyPudlak syndrome.

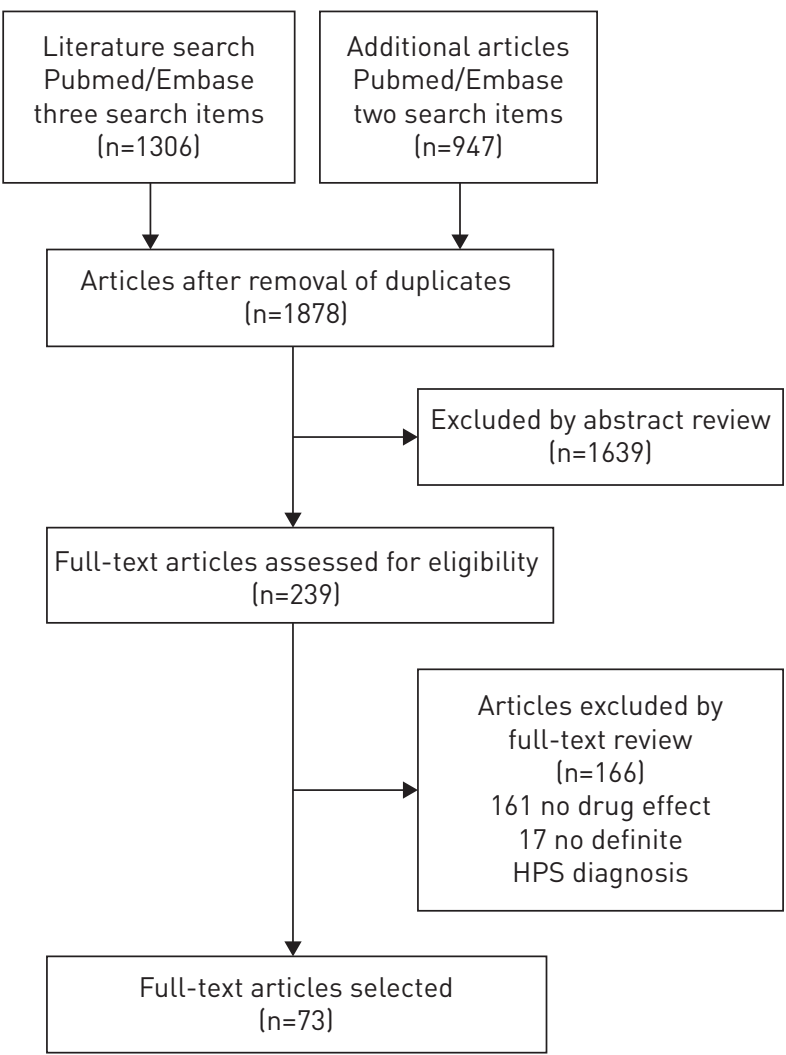


TABLE 1 Included studies on surfactant-processing mutations categorised by study type

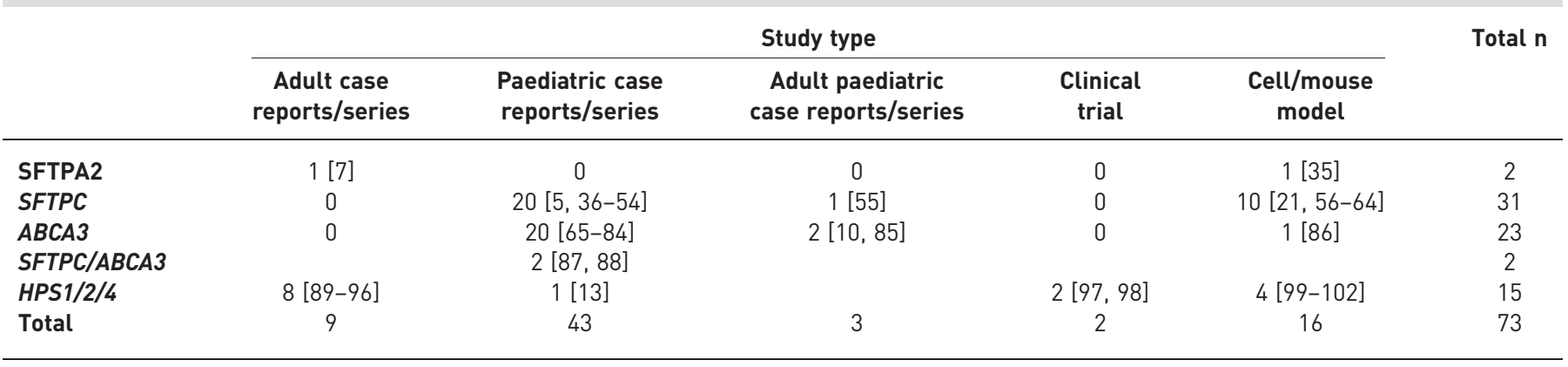

Data are presented as number of studies and their corresponding references.

other therapies. 55 case reports/series were included, of which 43 described paediatric cases, nine described adult cases and three described paediatric as well as adult cases. Adult patients were treated with corticosteroids, cyclosporine A, antibiotics, pirfenidone and/or azathioprine. Additional drugs used in paediatric patients were exogenous surfactant and hydroxychloroquine. Two clinical trials were included in which patients were treated with pirfenidone (table 2). The same drugs were tested in cell and mouse models (table 3). Additionally, drugs tested in cell and mouse models were glycerol, rapamycin, 4-phenylbutyric acid (PBA), saralasin, angiotensin (ANG)1-7, cyclophosphamide, recombinant CHI3L1, interleukin (IL) $13 \mathrm{R} \alpha 2$ construct, antibodies against monocyte chemotactic protein (MCP)-1 or SP-D and specific inhibitors for c-Jun N-terminal kinases (JNK), caspase 4, ADAM metallopeptidase domain 17/ tumor necrosis factor- $\alpha$-converting enzyme (ADAM17/TACE), synoviolin and extracellular signal-regulated kinases (ERK1/2, P38, nuclear factor (NF)-אB and CRTH2).

Human studies reported clinical symptoms, lung function, radiological characteristics or oxygen requirement as effect parameters. A majority of the cell-line and mouse studies investigated alterations in processes involved in surfactant trafficking [21, 35, 56-58], cytokine/chemokine concentrations [59, 60, 86, 99] and necrosis/apoptosis $[21,57,61-63,100]$, while some studies investigated weight loss, airway compliance [60], collagen secretion/accumulation [64, 100], migration of macrophages [101] or mortality [102] (table 3 and figure 2).

\section{Outcome in case reports/series and clinical trials}

An extensive description of the effect of treatment in paediatric and adult cases with a surfactant-processing mutation is described in online supplementary table S1. A concise outcome after treatment per drug combination (no effect, (short-term) improvement, (short-term) stabilisation, little effect) was determined based on the information derived from case reports/series. In addition, an overall outcome after treatment was determined based on the last reported outcome after treatment with different drugs and displayed in table 2. In six of the 12 adult case studies (five patients in total) $[7,10,85,89-91]$, (short-term) improvement or (short-term) stabilisation of the disease was observed after treatment with pirfenidone, corticosteroids or antibiotics. In paediatric case studies (short-term) stabilisation or (short-term) improvement of disease was observed in 20 of the 23 case reports/series [5, 36-51, 87, 88, 103] (in five case series, not in all described cases; total 67 patients) describing patients with a SFTPC mutation treated with hydroxychloroquine, surfactant, antibiotics or corticosteroids. In addition, (short-term) improvement or (short-term) stabilisation of disease was found in 15 of the 24 case reports/ series describing paediatric cases $[10,65-76,85,88]$ (in four case series, not in all described cases; 28 patients in total) describing patients with an $A B C A 3$ mutation treated with the same drugs. In one case report describing a drug effect of corticosteroids in a patient with an AP3B1 mutation, stabilisation of disease was reported [13]. One of the two clinical trials included in this review [97] reported a positive effect of pirfenidone on lung function of HPS patients with a baseline forced vital capacity (FVC) $\geqslant 50 \%$ predicted. The other clinical trial reported no statistically significant difference between pirfenidone and the placebo group [98].

\section{Outcome in mouse studies and cells derived from humans with an HPS1 mutation}

The effect of drugs on mouse or human lung cells with an HPS1 mutation was investigated in three studies [99-101]. Treatment of alveolar macrophages from bronchoalveolar lavage (BAL) fluid derived from patients with an HPS1 mutation with pirfenidone resulted in reduced cytokine/chemokine secretion [99]. In addition, changes in macrophage behaviour were found in experiments with BAL fluid from 
TABLE 2 Case reports/series and clinical trials of humans with a surfactant-processing mutation involved in fibrotic interstitial pneumonia

Outcome after Overall outcome

after treatment

[Ref.]

reatment per drug

No effect

Adult with CPFE

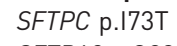

SFTPC p.173T

SFTPA2 p.G231V

ABCA3 p.G964D

ABCA3 p.G964D

HPS

HPS1 IVS5+5 G>A

HPS4 p.Q620X

HPS1 p.L668P

HPS ${ }^{\#}$

HPS4 p.685delC

HPS $^{\#}$

HPS

Paediatric case reports/series with SFTPC mutations SFTPC p.I73T p.I38F, p.V39L

SFTPC p.A116D

SFTPC p.I73T

SFTPC c.460+1 G $\rightarrow$ A

SFTPC p.I73T

Different SFTPC BRICHOS/non-BRICHOS

SFTPC 14 non-BRICHOS, 6 BRICHOS
1 adult with hypersensitivity pneumonitis

1 adult with pulmonary

fibrosis and bronchoalveolar carcinoma

$$
\begin{aligned}
& \text { Adult with pulmonary } \\
& \text { fibrosis }
\end{aligned}
$$

Adult with restrictive lung disease

Adult with HPS

Adult with HPS

Adult with HPS

Adult with HPS

Adult with HPS

Adult with HPS

Adult with HPS and

pulmonary sarcoidosis

Adult with HPS

4 children with DIP, 1 child with chronic interstitial pneumonitis (p.V39L)

$$
\begin{aligned}
& \text { Child with NSIP } \\
& \text { Child with ILD }
\end{aligned}
$$

Child with cellular or NSIP

5 children with chronic ILD

17 children with ILD (NSIP, PAP or DIP)

22 children with chronic ILD at diagnosis
Prednisone and avoidance of birds

Prednisone

Antibiotics

Prednisone and azithromycin

Steroids and azithromycin

Antibiotics and oxygen inhalation

Prednisolone, cyclosporine A

$$
+ \text { pirfenidone }
$$

Corticosteroids, pirfenidone

Prednisolone, pirfenidone, azathioprine Oral corticosteroids

High-dose steroids and azathioprine Prednisone

Prednisolone and pirfenidone

$$
1 / 5 \text { systemic steroids }
$$$$
5 / 5 \text { hydroxychloroquine }
$$

Hydroxychloroquine and supplemental oxygen Corticosteroids and supplemental oxygen

Corticosteroids and supplemental oxygen $5 / 5$ methylprednisolone, $4 / 5$ hydroxychloroquine, $5 / 5$ supplemental oxygen 14/17 hydroxychloroquine

15/17 systemic steroids

$7 / 17$ surfactant

$3 / 17$ colchicine

18/22 methylprednisolone,

11/22 hydroxychloroquine, 5/22 azithromycin

20/22 supplemental oxygen
No effect

Improvement

No effect

No effect

Stabilisation

(Short-term)

improvement

No effect

No effect

Short-term stabilisation

Stabilisation

No effect

No effect

No effect

Improvement

No effect

No effect

2 short-term

improvement, 3

improvement

Improvement

Improvement

Improvement

5 improvement

12/14 improvement

2/14 no effect

14/15 improvement

$1 / 15$ no effect

2/7 improvement

$5 / 7$ no effect

$6 / 22$ no effect,

16/22 improvement improvement/

1 no effect

Stabilisation

Stabilisation

No effect

Short-term

stabilisation

Stabilisation

No effect

No effect

No effect

Improvement

No effect

2 short-term

improvement/3

improvement

Improvement

Improvement

5 improvement

7 improvement/

7 stabilisation/

3 no effect

[90]

[93]

[95

[37]

[55] 


\begin{tabular}{|c|c|c|c|c|c|}
\hline & Diagnosis & Drug & $\begin{array}{l}\text { Outcome after } \\
\text { treatment per drug } \\
\text { combination }\end{array}$ & $\begin{array}{l}\text { Overall outcome } \\
\text { after treatment }\end{array}$ & [Ref.] \\
\hline SFTPC p.G97S & $\begin{array}{l}\text { Child with CPI pattern with } \\
\text { globular alveolar proteinosis }\end{array}$ & $\begin{array}{l}\text { Home ventilator support, oxygen, pulse } \\
\text { methylprednisolone, azithromycin, } \\
\text { hydroxychloroquine }\end{array}$ & Improvement & Improvement & [41] \\
\hline SFTPC p.I73T, p.I38F & 2 children with $\mathrm{CPI}$ & $\begin{array}{l}\text { Hydroxychloroquine, prednisone, ranitidine, } \\
\text { TMP-SMX; } 2 \text { months after therapy began, } \\
\text { pulse therapy of methylprednisolone; later, } \\
\text { hydroxychloroquine alone }\end{array}$ & 2 improvement & 2 improvement & [42] \\
\hline SFTPC p.I73T & $\begin{array}{l}\text { Child with CPI, } \\
\text { pneumatoceles after biopsy }\end{array}$ & $\begin{array}{l}\text { Methylprednisolone, hydroxychloroquine, } \\
\text { azithromycin }\end{array}$ & Improvement & Improvement & [43] \\
\hline \multirow[t]{3}{*}{ SFTPC p.I73T } & $\begin{array}{l}\text { Child with ILD } \\
\text { Child with ARDS/DIP }\end{array}$ & $\begin{array}{l}\text { Hydroxychloroquine, oxygen supplementation } \\
\text { Supplemental oxygen and steroids } \\
\text { Steroids pulse therapy }\end{array}$ & $\begin{array}{l}\text { Improvement } \\
\text { No effect } \\
\text { Short-term } \\
\text { improvement }\end{array}$ & $\begin{array}{l}1 \text { improvement/ } \\
1 \text { stabilisation/ } \\
1 \text { no effect }\end{array}$ & [44] \\
\hline & & Hydroxychloroquine & Improvement & & \\
\hline & Child with DIP & $\begin{array}{l}\text { Hydroxychloroquine replaced by azithromycin } \\
\text { Hydroxychloroquine and steroids }\end{array}$ & $\begin{array}{l}\text { Stabilisation } \\
\text { No effect }\end{array}$ & & \\
\hline SFTPC p.I73T & Child with chILD & $\begin{array}{l}\text { Bronchodilators, inhaled corticosteroids and } \\
\text { antileukotrienes, azathioprine, } \\
\text { hydroxychloroquine and i.v. immunoglobulins, } \\
\text { exogenous surfactant }\end{array}$ & No effect & No effect & [52] \\
\hline SFTPC p.I73T & Child with PAP and NSIP & $\begin{array}{c}\text { Supplemental oxygen, whole-lung lavages, } \\
\text { systemic corticosteroids and azathioprine } \\
\text { Additional corticosteroid pulse therapy plus } \\
\text { azathioprine }\end{array}$ & $\begin{array}{l}\text { Short-term } \\
\text { improvement } \\
\text { No effect }\end{array}$ & $\begin{array}{l}\text { Short-term } \\
\text { improvement }\end{array}$ & [45] \\
\hline SFTPC $\triangle$ exon 4 & $\begin{array}{l}\text { Child with respiratory } \\
\text { distress }\end{array}$ & $\begin{array}{l}\text { Oral and i.v. corticosteroids, } \\
\text { hydroxychloroquine, supplemental oxygen }\end{array}$ & Improvement & Improvement & [51] \\
\hline \multirow[t]{6}{*}{ SFTPC p.E66K, p.I73T, p.V102M, p.A155P } & $\begin{array}{l}8 \text { children with idiopathic } \\
\text { diffuse lung diseases }\end{array}$ & $\begin{array}{l}2 \text { children supplemental oxygen, pulse } \\
\text { steroids and hydroxychloroquine }\end{array}$ & 2 limited effect & $\begin{array}{l}2 \text { limited effect } / 6 \\
\text { stabilisation }\end{array}$ & [46] \\
\hline & & $\begin{array}{l}\text { Supplemental oxygen, pulse steroids, } \\
\text { hydroxychloroquine }\end{array}$ & Stabilisation & & \\
\hline & & Supplemental oxygen, hydroxychloroquine & Stabilisation & & \\
\hline & & $\begin{array}{l}\text { Supplemental oxygen, steroids, } \\
\text { hydroxychloroquine, azithromycin }\end{array}$ & Stabilisation & & \\
\hline & & $\begin{array}{l}\text { Supplemental oxygen, pulse steroids, } \\
\text { hydroxychloroquine }\end{array}$ & Stabilisation & & \\
\hline & & $\begin{array}{l}\text { Supplemental oxygen, pulse steroids, } \\
\text { bronchodilators, antibiotics }\end{array}$ & Stabilisation & & \\
\hline SFTPC p.L188Q & $\begin{array}{l}2 \text { children with respiratory } \\
\text { distress (NSIP-like pattern) }\end{array}$ & $\begin{array}{l}\text { Steroids, supplemental oxygen } \\
\text { Methylprednisolone and hydroxychloroquine }\end{array}$ & $\begin{array}{l}\text { Stabilisation } \\
2 \text { no effect }\end{array}$ & 2 no effect & [53] \\
\hline SFTPC \# & Child with CPI & $\begin{array}{l}\text { Corticosteroids, hydroxychloroquine and } \\
\text { continuous oxygen }\end{array}$ & $\begin{array}{l}\text { Short-term } \\
\text { improvement }\end{array}$ & $\begin{array}{l}\text { Short-term } \\
\text { improvement }\end{array}$ & [47] \\
\hline
\end{tabular}




\begin{tabular}{|c|c|c|c|c|c|}
\hline & Diagnosis & Drug & $\begin{array}{l}\text { Outcome after } \\
\text { treatment per drug } \\
\text { combination }\end{array}$ & $\begin{array}{l}\text { Overall outcome } \\
\text { after treatment }\end{array}$ & [Ref.] \\
\hline SFTPC p.I73T & Child with NSIP/PAP & $\begin{array}{l}\text { Supplemental oxygen, antibiotics and oral } \\
\text { corticosteroids }\end{array}$ & No effect & No effect & [54] \\
\hline SFTPC p.I73T & Child with PAP/ILD & $\begin{array}{l}\text { Whole-lung lavages, systemic corticosteroids } \\
\text { and azathioprine }\end{array}$ & Improvement & Improvement & [48] \\
\hline \multirow[t]{3}{*}{ SFTPC p.G182R, p.L188Q, p.C189W } & 1 child with PAP/NSIP & $\begin{array}{c}\text { Clearance, steroids, hydroxychloroquine, } \\
\text { mechanical ventilation }\end{array}$ & Improvement & 3 improvement & [49] \\
\hline & $\begin{array}{l}1 \text { child with respiratory } \\
\text { failure }\end{array}$ & $\begin{array}{c}\text { Clearance, steroids, azathioprine, mechanical } \\
\text { ventilation }\end{array}$ & Improvement & & \\
\hline & $\begin{array}{l}1 \text { child with respiratory } \\
\text { failure }\end{array}$ & $\begin{array}{l}\text { Steroids, azithromycin, hydroxychloroquine, } \\
\text { mechanical ventilation }\end{array}$ & Improvement & & \\
\hline SFTPC p.L81V & $\begin{array}{l}\text { Child with surfactant protein } \\
\qquad \text { C deficiency }\end{array}$ & Hydroxychloroquine, oxygen therapy & Improvement & Improvement & [87] \\
\hline SFTPC $\#$ & Child with NSIP & $\begin{array}{l}\text { Systemic steroids, azathioprine, } \\
\text { hydroxychloroquine }\end{array}$ & Improvement & Improvement & [88] \\
\hline Different SFTPC mutations & $\begin{array}{l}15 \text { children with interstitial } \\
\text { chronic lung disease }\end{array}$ & $\begin{array}{l}\text { All methylprednisolone } \\
5 / 15 \text { azithromycin } \\
8 / 15 \text { hydroxychloroquine }\end{array}$ & $\begin{array}{l}11 \text { no effect/ } \\
4 \text { improvement }\end{array}$ & $\begin{array}{l}11 \text { no effect/ } \\
4 \text { improvement }\end{array}$ & [50] \\
\hline \multicolumn{6}{|c|}{ Paediatric case reports/series with $A B C A 3$ mutations } \\
\hline$A B C A 3^{\#} / S F T P C$ & $\begin{array}{c}\text { Child with DPLD/surfactant } \\
\text { dysfunction }\end{array}$ & $\begin{array}{l}\text { Surfactant } \\
\text { Systemic steroids }\end{array}$ & $\begin{array}{l}\text { Improvement } \\
\text { No effect }\end{array}$ & $\begin{array}{l}2 \text { short-term } \\
\text { improvement/ } \\
1 \text { no effect }\end{array}$ & [88] \\
\hline \multirow[t]{3}{*}{$A B C A 3^{\#}$} & Child with CPI & Systemic steroids, surfactant & Improvement & & \\
\hline & & Chloroquine & No effect & & \\
\hline & Child with DIP & Systemic steroids, hydroxychloroquine & No effect & & \\
\hline ABCA3 c.358_359del & Child with $A B C A 3$ deficiency & Methylprednisolone, oxygen therapy & Limited effect & Limited effect & [87] \\
\hline \multirow[t]{3}{*}{ ABCA3 p.W1148X and p.T1114A } & Child with PAP-like features & $\begin{array}{l}\text { Methylprednisolone, antibiotics, antivirals and } \\
\text { antifungals, oxygen, mechanical ventilation }\end{array}$ & No effect & Improvement & {$[76]$} \\
\hline & & BAL with bovine surfactant & Improvement & & \\
\hline & & Hydroxychloroquine & Improvement & & \\
\hline ABCA3 p.G964D & Child with (possible) IPF & Prednisone and macrolides & Improvement & Improvement & [10] \\
\hline \multirow[t]{2}{*}{$A B C A 3$ p.A307V } & $\begin{array}{l}\text { Child with respiratory } \\
\text { distress }\end{array}$ & Dexamethasone and surfactant, CPAP & $\begin{array}{l}\text { Short-term } \\
\text { improvement }\end{array}$ & Improvement & [75] \\
\hline & & $\begin{array}{l}\text { Methylprednisolone, azithromycin, } \\
\text { hydroxychloroquine }\end{array}$ & Improvement & & \\
\hline ABCA3 p.Y1515X & Child with RDS & Pulse steroids, antibiotics & No effect & No effect & [77] \\
\hline ABCA3 p.R194G and V1615GfsX15 & 2 children with IRDS & CPAP, corticosteroids and hydroxychloroquine & 2 limited effect & 2 limited effect & [78] \\
\hline \multirow[t]{2}{*}{ ABCA3 p.D253H } & Child with DPLD & $\begin{array}{c}\text { Methylprednisolone, oral prednisone, } \\
\text { oxygen therapy }\end{array}$ & No effect & Improvement & [74] \\
\hline & & Azithromycin & Improvement & & \\
\hline \multirow[t]{2}{*}{$A B C A 3$ p.R280C and p.E690G } & Child with DIP & $\begin{array}{l}\text { Oxygen supplementation, surfactant therapy, } \\
\text { corticosteroids }\end{array}$ & No effect & Improvement & [73] \\
\hline & & Hydroxychloroquine & Improvement & & \\
\hline
\end{tabular}




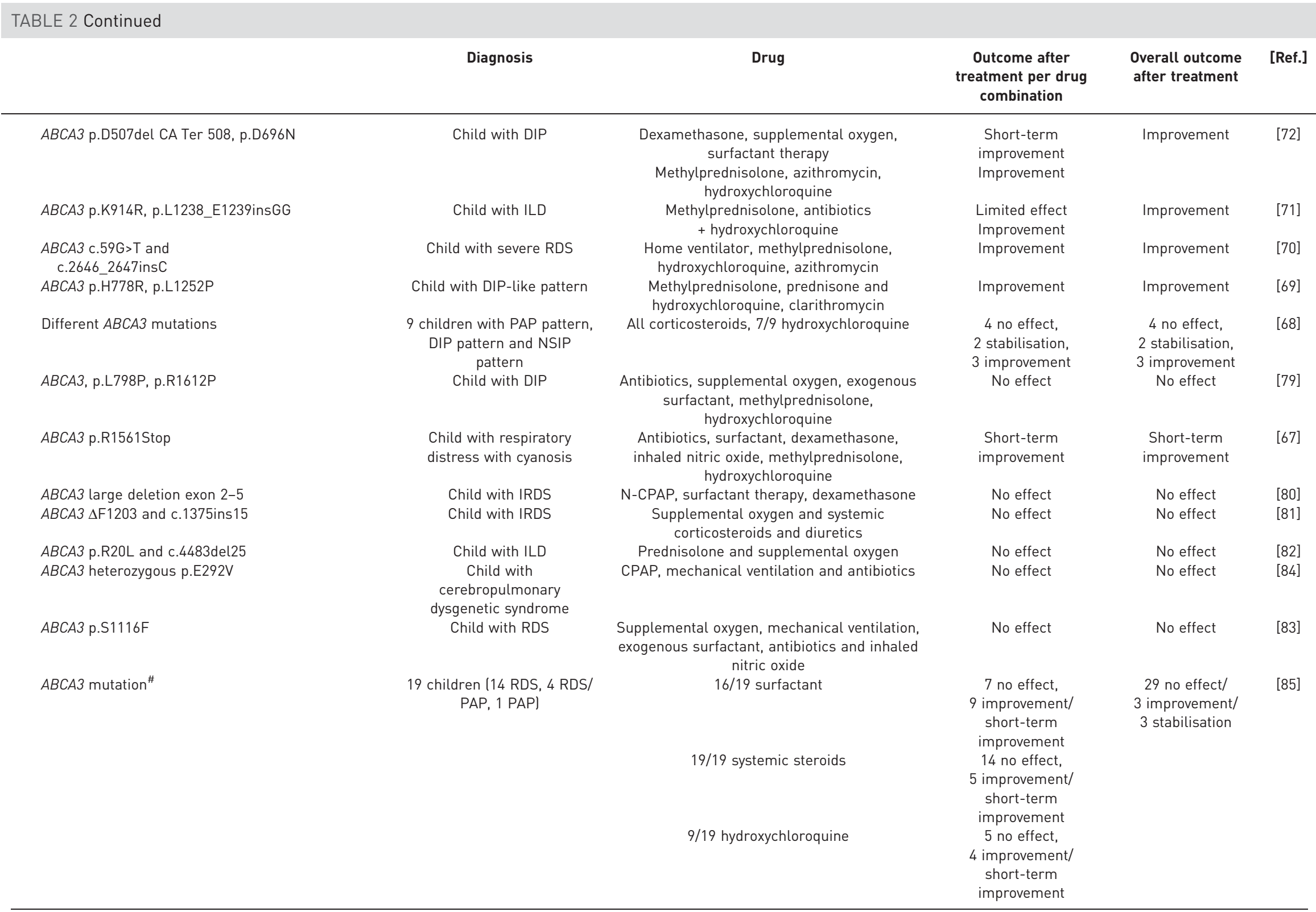


TABLE 2 Continued

\begin{tabular}{|c|c|c|c|c|c|}
\hline & Diagnosis & Drug & $\begin{array}{l}\text { Outcome after } \\
\text { treatment per drug } \\
\text { combination }\end{array}$ & $\begin{array}{l}\text { Overall outcome } \\
\text { after treatment }\end{array}$ & [Ref.] \\
\hline \multirow[t]{2}{*}{ Heterozygous $A B C A 3$ mutation" } & $\begin{array}{l}16 \text { children }(9 \text { with RDS, } 4 \\
\text { with RDS/PAP, } 1 \text { PAP, } 2 \\
\text { chlLD) }\end{array}$ & $\begin{array}{l}\text { 2/19 azithromycin } \\
12 / 16 \text { surfactant }\end{array}$ & $\begin{array}{l}2 \text { no effect } \\
8 \text { no effect, } 4 \\
\text { improvement/short } \\
\text { term improvement }\end{array}$ & & \\
\hline & & $12 / 16$ systemic steroids & $\begin{array}{l}8 \text { no effect, } \\
4 \text { improvement/ } \\
\text { short-term } \\
\text { improvement }\end{array}$ & & \\
\hline \multirow[t]{2}{*}{$\begin{array}{l}A B C A 3 \text { p.M1227R and } \\
\text { Ins1510fs/ter1519 }\end{array}$} & Child with DIP & $\begin{array}{l}\text { Macrolides, dexamethasone, } \\
\text { mechanical ventilation }\end{array}$ & No effect & $\begin{array}{l}\text { Short-term } \\
\text { improvement }\end{array}$ & [66] \\
\hline & & Surfactant & $\begin{array}{l}\text { Short-term } \\
\text { improvement }\end{array}$ & & \\
\hline $\begin{array}{l}\text { ABCA3 heterozygous R288K (7 patients) p.R43L, } \\
\text { R288K + c.4751delT (1 patient), R288K, P766S } \\
\text { (heterozygous, } 1 \text { patient), R288K, S693L } \\
\text { (heterozygous, } 1 \text { patient), R288K, Q215K (1 patient) }\end{array}$ & 11 children with ILD & $\begin{array}{l}\text { 5/11 prednisolone, surfactant, oxygen or } \\
\text { corticosteroids } \\
\text { 6/11 oxygen, aspirin, surfactant, } \\
\text { dexamethasone, montelukast, salbutamol, } \\
\text { steroids, hydroxychloroquine, azathioprine, } \\
\text { azithromycin or antibiotics }\end{array}$ & $\begin{array}{l}\text { No effect } \\
\text { Improvement }\end{array}$ & $\begin{array}{l}5 \text { no effect/ } \\
6 \text { improvement }\end{array}$ & [65] \\
\hline $\begin{array}{l}A P 3 B 1 p . R 509 X \text { and } p . E 659 X \\
\text { Clinical trials } \\
\quad H P S 1^{11}\end{array}$ & 21 adults with HPS & 11 treated with pirfenidone, 10 placebo & \multicolumn{2}{|c|}{$\begin{array}{c}\text { Pirfenidone superior to placebo: } \Delta F V C \text { of } \\
0.46 \% \text { per month ( } p=0.587) \\
\text { Restricted group including only patients } \\
\text { with initial FVC values }>50 \% \text { pred: } \\
\text { difference in pulmonary function } \sim 0.7 \% \text { per } \\
\text { month ( } p=0.02 \text { ) }\end{array}$} & [97] \\
\hline HPS1 or $4^{+}$ & 35 adults with HPS & 23 treated with pirfenidone, 12 placebo & $\begin{array}{r}\text { No statistically signifi } \\
\text { fun }\end{array}$ & difference in lung & [98] \\
\hline
\end{tabular}

ABCA3 mutations were compound heterozygous or homozygous mutations, unless otherwise stated. CPFE: combined pulmonary fibrosis and emphysema; HPS: Hermansky-Pudlak syndrome; DIP: desquamative interstitial pneumonia; NSIP: nonspecific interstitial pneumonia; ILD: interstitial lung disease; PAP: pulmonary alveolar proteinosis; CPI: chronic pneumonitis of infancy; ARDS: acute respiratory distress syndrome; chILD: childhood ILD; DPLD: diffuse parenchymal lung disease; BAL: bronchoalveolar lavage; IPF: interstitial pulmonary fibrosis; (N)-CPAP: (nasal)-continuous positive airway pressure; RDS: respiratory distress syndrome; IRDS: infant respiratory distress syndrome; G-CSF: granulocyte colony-stimulating factor; $\triangle \mathrm{FVC}$ : change in forced vital capacity. ${ }^{\#}$ : specific mutation not mentioned in the article, diagnosis based on absence of platelet dense bodies under electron microscopy or genetic testing; ${ }^{\Uparrow}: 20$ of these patients were Puerto Ricans homozygous for a 16-bp duplication in exon 15 of the HPS1 gene, which leads to a frameshift. The other patient was a Puerto Rican with a 3904-bp deletion in the HPS3 gene; ${ }^{+}: 33$ of these patients were Puerto Ricans homozygous for the known 16 -bp duplication in exon 15 of the HPS 1 gene. Two patients were non-Puerto Rican, and the mutations in these patients are not reported. 


\section{TABLE 3 Drug effect in cell and mouse models with a surfactant-processing mutation}

\section{Effect paramete}

Chaperone protein expression

$\mathrm{LDH}$ release

(Hydroxy)chloroquine

Accumulation SP-C proprotein

SP-C mature protein

Mislocalisation defect proSP-C

Chaperone protein

HSP70 protein expression ${ }^{\#}$

LDH release

LPC and PC levels

Methylprednisolone

Chaperone protein expression ${ }^{\#}$

HSP70 protein expression"

$\mathrm{LDH}$ release

Mislocalisation defect proSP-C

$P C$ and LPC levels

Cyclophosphamide
Outcome after treatment

Calnexin, calreticulin, HSP70: no effect ${ }^{\#}$ HSP90: increased ${ }^{\#}$

Further increased

Increased

$$
\text { Increased }
$$

No significant effect

No significant effect

No significant effect

No significant chang

Calnexin: no effect

Calreticulin, HSP90: increased

$$
\text { Increased }
$$$$
\text { No effect }
$$

No effect on increased levels

$$
\text { Increased }
$$

Intracellular: no correction loss of PC

$$
\text { amelioration of LPC increase }
$$

Supernatant: amelioration of the reduction in PC

but no significant effect on increased

$$
\text { LPC }
$$

Intracellular: amelioration of reduced PC,

$$
\text { reduction in increased LPC }
$$

Supernatant: no effect on PC, restored LPC

Calnexin, calreticulin: no effect

$$
\begin{gathered}
\text { HSP9O increase } \\
\text { Increased } \\
\text { No effect }
\end{gathered}
$$$$
\text { No effect }
$$

Partial correction of reduced proSP-C in secretory vesicles and increased proSP-C in

$$
\text { early endosomal vesicles }
$$

Intracellular: no correction of loss of PC.

amelioration of increased LPC

Supernatant: no effect on PC and no effect on

$$
\text { increased LPC }
$$

Intracellular: no correction loss of PC amelioration of LPC increase

Supernatant: amelioration of the reduction in PC but no significant effect on increased LPC

$$
\text { Calnexin: no effect }{ }^{\#}
$$

Calreticulin, HSP70, HSP90: increased No effect on increased levels

$$
\text { No effect }
$$

Model system

Gene mutation

[Ref.]

$\begin{array}{ccc}\text { MLE12 } & \text { SFTPC p.A116D, } & {[21,} \\ & \text { p.I73T } & 57] \\ \text { MLE12 } & \text { SFTPC p.A116D } & {[57]} \\ & \text { SFTPC p.I73T } & {[21]} \\ \text { SE12, HEK293 } & \text { SFTPC p.L188Q } & {[56]} \\ \text { HEK293 } & \text { SFTPC p.I73T, } \\ & \Delta \text { exon4 } \\ \text { HEK293 } & \text { SFTPC p.L188Q, } \\ & \text { p.I73T, } \Delta \text { exon4 } \\ \text { MLE12 } & \text { SFTPC p.L188Q } \\ \text { MLE12 } & \text { SFTPC p.173T } & {[21]} \\ \text { MLE12 } & \text { SFTPC p.A116D } & {[21,} \\ & \text { p.I73T } & 57] \\ & \text { SFTPC p.A116D } & {[57]} \\ \text { MLE12 } & \text { SFTPC p.I73T } & {[21]} \\ & \text { SFTPC p.A116D } & {[57]} \\ \text { MLE12 } & \text { SFTPC p.I73T } & {[21]} \\ & \text { SFTPC p.I73T } & {[21]}\end{array}$

SFTPC p.A116D

MLE12

SFTPC p.A116D,

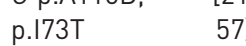
SFTPC p.A116D [57] SFTPC $\mathrm{p} .173 \mathrm{~T}$ SFTPC p.A116D, [21 SFTPC p.I73T 57] SFTPC p.I73T [21]

MLE12

SFTPC p.A116D [57]

SFTPC p.I73T

MLE12

$\begin{array}{cc}\text { SFTPC p.A116D, } & \text { [21, } \\ \text { p.I73T } & 57] \\ \text { SFTPC p.A116D } & {[57]} \\ \text { SFTPC p.I73T } & \text { [21] }\end{array}$




\begin{tabular}{|c|c|c|c|c|c|}
\hline & Effect parameter & Outcome after treatment & Model system & Gene mutation & [Ref.] \\
\hline Rapamycin & $\begin{array}{c}\text { Airway compliance, weight loss } \\
\text { IL-4, IL-13 gene expression } \\
\text { Total lung collagen, IFN- } \gamma \text { gene } \\
\text { expression, airway resistance } \\
\text { Total BALF cells }\end{array}$ & $\begin{array}{c}\text { Further reduced } \\
\text { Further increased } \\
\text { No effect on increased levels } \\
\text { Attenuation of increase }\end{array}$ & $\begin{array}{l}\text { 129S6/Sv Bleomycin-treated } \\
\text { mice }\end{array}$ & SFTPC-/- & {$[60]$} \\
\hline $\begin{array}{l}\text { Ascorbate or anti-SP-D } \\
\text { and/or anti-MCP-1 }\end{array}$ & Migration of RAW 264.7 cells & Amelioration of increase & $\begin{array}{l}\text { BAL from EPPE C57BL/6J } \\
\text { mice and HPS1 patients }\end{array}$ & HPS1 & [101] \\
\hline \multirow[t]{17}{*}{ 4-phenylbutyric acid } & $\begin{array}{l}\text { Accumulation of detergent } \\
\text { insoluble aggregates }\end{array}$ & $\begin{array}{l}\text { Further increased } \\
\text { No change in increase } \\
\text { Slightly increased }\end{array}$ & HEK293 & $\begin{array}{l}\text { SFTPC p.L188Q } \\
\text { SFTPC p.I73T } \\
\text { SFTPC } \Delta \text { exon4 }\end{array}$ & {$[56]$} \\
\hline & & $\begin{array}{l}\text { NP-40 insoluble aggregates: slight attenuation of } \\
\text { increase }\end{array}$ & $\mathrm{CHO}-\mathrm{K} 1$ & SFTPA2 p.G231V & [35] \\
\hline & & $\begin{array}{l}\text { NP-40 insoluble aggregates: amelioration of } \\
\text { increase }\end{array}$ & & SFTPA2 p.F198S & \\
\hline & Accumulation of SP-C proprotein & $\begin{array}{l}\text { No significant change in increase } \\
\text { Attenuation of reduction }\end{array}$ & HEK 293 & $\begin{array}{c}\text { SFTPC p.I73T } \\
\text { SFTPC p.L188Q, } \\
\Delta \text { exon4 }\end{array}$ & {$[56]$} \\
\hline & $\begin{array}{l}\text { Juxtanuclear mutant SP-C } \\
\text { accumulation }\end{array}$ & Corrected & A549 & SFTPC $\triangle$ exon4 & {$[58]$} \\
\hline & $\mathrm{SP}-\mathrm{C}$ mature protein & $\begin{array}{l}\text { Increased to WT concentrations } \\
\text { Amelioration of reduction } \\
\text { No effect on reduced expression }\end{array}$ & HEK293 & $\begin{array}{l}\text { SFTPC p.L188Q } \\
\text { SFTPC p.I73T } \\
\text { SFTPC } \Delta \text { exon4 }\end{array}$ & {$[56]$} \\
\hline & Mutant SP-A2 protein secretion & Partial attenuation of reduction & $\mathrm{CHO}-\mathrm{K} 1$ & $\begin{array}{l}\text { SFTPA2 p.G231, } \\
\text { p.F198S }\end{array}$ & [35] \\
\hline & ER stress & $\begin{array}{l}\text { Increased } \\
\text { No effect }\end{array}$ & HEK293 & $\begin{array}{c}\text { SFTPC p.L188Q } \\
\text { SFTPC } \triangle \text { exon4 and } \\
\text { p.I73T }\end{array}$ & {$[56]$} \\
\hline & $\begin{array}{l}\text { ER stress-induced factors } \\
\text { (spliced XBP1, ATF6, } \\
\text { cathepsin D) }\end{array}$ & Attenuation of increase & A549 & SFTPC p.G100S & [63] \\
\hline & Phosphorylation of elF2 $\alpha$ & Attenuation of increase & A549 & SFTPC p.G100S & \\
\hline & Caspase 3 activation & Attenuation of increase & A549 & SFTPC p.G100S & \\
\hline & Nuclear fragmentation & Attenuation of increase & A549 & SFTPC p.G100S & \\
\hline & ADAM17/TACE levels & Attenuation of increase & A549 & SFTPC p.G100S & \\
\hline & $N F-\kappa B$ induction & Amelioration of increase & A549 & SFTPC $\triangle$ exon4 & [59] \\
\hline & Phosphorylated JNK and AP-1 & Hardly any change in increased expression & A549 & SFTPC $\triangle$ exon4, & \\
\hline & expression & Stimulated & HEK293 & p.L188Q & \\
\hline & IL-8 concentration & Enhanced & A549 & SFTPC $\triangle$ exon 4 & \\
\hline \multicolumn{6}{|l|}{$\begin{array}{l}\text { Anti-fibrotic/ } \\
\text { immunosuppressive agent }\end{array}$} \\
\hline \multirow[t]{2}{*}{ Pirfenidone } & $\begin{array}{l}\text { GM-CSF and IL-12p40 } \\
\text { expression }\end{array}$ & No effect on increased levels, & $\begin{array}{l}\text { Alveolar macrophages from } \\
\text { BALF of HPS1 subjects }\end{array}$ & HPS1 & [99] \\
\hline & $\begin{array}{l}\text { MIP- } 1 \alpha, \text { MCP- } 1, \text { RANTES, } \\
\text { M-CSF, MIP- } 4 \text { and IFN- } \gamma\end{array}$ & Amelioration of increase & & & \\
\hline
\end{tabular}




\section{MAPK signalling pathway}

inhibitors
ERK $1 / 2$ inhibitor
IL-8 secretion
P38 inhibitor
IL-8 secretion
SP600125 ( JNK inhibitor)
IL-8 concentration

\section{Antibiotics}

Bafilomycin A1/azithromycin Accumulation SP-C proprotein

SP-C mature protein

Combination therapy

4-phenylbutyric acid + SP600125 ( JNK inhibitor) SP600125 ( JNK inhibitor) and/or caspase 4 inhibitor

\section{Anti-apoptotic therapy}

Pan-caspase inhibitor

\section{Other drugs}

TAPI-2 (ADAM17/TACE-specific inhibitor)

Saralasin (ANGII receptor

antagonist), synthetic ANG1-7

Glycerol

LS-102 (synoviolin inhibitor)

$\mathrm{NF}-\mathrm{\kappa B}$ inhibitor

Recombinant CHI3L1

IL-13R $\alpha 2$ construct

CRTH2 inhibitor
IL-8 concentration

Activation of caspase 3 cleavage

DNA fragmentation

Mortality

ACE-2 loss

Increase in nuclear

fragmentation ${ }^{\#}$

SP-C concentration

Collagen secretion

IL-8 secretion

Cell apoptosis

Cell apoptosis

Collagen accumulation
Attenuation of increase No effect

Completely inhibited the increase in concentration

No effect

No significant effect

Increased

No significant effect

No significant effect

Completely antagonised

Amelioration of increase

Amelioration of increase

Amelioration of increase

Reduced to WT levels

Unchanged

Attenuation of increase

No effect

No effect

Amelioration of increase

Amelioration of increase
A549

A549

A549

A549

HEK293

MLE12, HEK293

HEK293

MLE12

A549

HEK293

HEK293/A549

Bleomycin challenged C57BL/ 6J mice

A549

A549

HEK293

A549

A549

Bleomycin-treated AEC2 from pale ear C57BL/6 mice

Bleomycin-treated AEC2 from pale ear C57BL/6 mice

Bleomycin-treated AEC2 from pale ear C57BL/6 mice
ABCA3 p.T1173R [86]

ABCA3 p.T1173R [86]

SFTPC $\triangle$ exon4 [59]

ABCA3 p.T1173R [86]

SFTPC p.173T,

$\triangle$ exon4

SFTPC p.L188Q

SFTPC p.L188Q,

p.I73T, $\triangle$ exon4

SFTPC p.L188Q

SFTPC $\triangle$ exon4

SFTPC $\triangle$ exon4,

p.L188Q

SFTPC $\triangle$ exon 4

HPS2 homozygous

SFTPC p.G100S

SFTPC p.G100S

SFTPC p.L188Q,

$\triangle$ exon4, p.173T

SFTPC $\triangle$ exon4 [64]

ABCA3 p.T1173R [86]

HPS1 null [100]

HPS1

HPS1

SP: surfactant protein; MCP: monocyte chemotactic protein; MAPK: mitogen-activated protein kinase; ERK: extracellular signal-regulated kinase; JNK: c-jun N-terminal kinase; ANG: angiotensin; NF: nuclear factor; IL: interleukin; HSP: heat shock protein; LDH: lactate dehydrogenase; LPC: lysophosphatidylcholine; PC: phosphatidylcholine; IFN: interferon; BAL: bronchoalveolar lavage; EPPE: Hps1ep/Hps1ep. Ap3b1pe/Ap3b1pe; BALF: bronchoalveolar lavage fluid; WT: wild type; ER: endoplasmic reticulum; GM-CSF: granulocyte-macrophage colony stimulating factor; MIP: macrophage inflammatory protein; ACE: angiotensin-converting enzyme; AEC2: alveolar type II cells. \#: outcome compared to untreated wild type. 


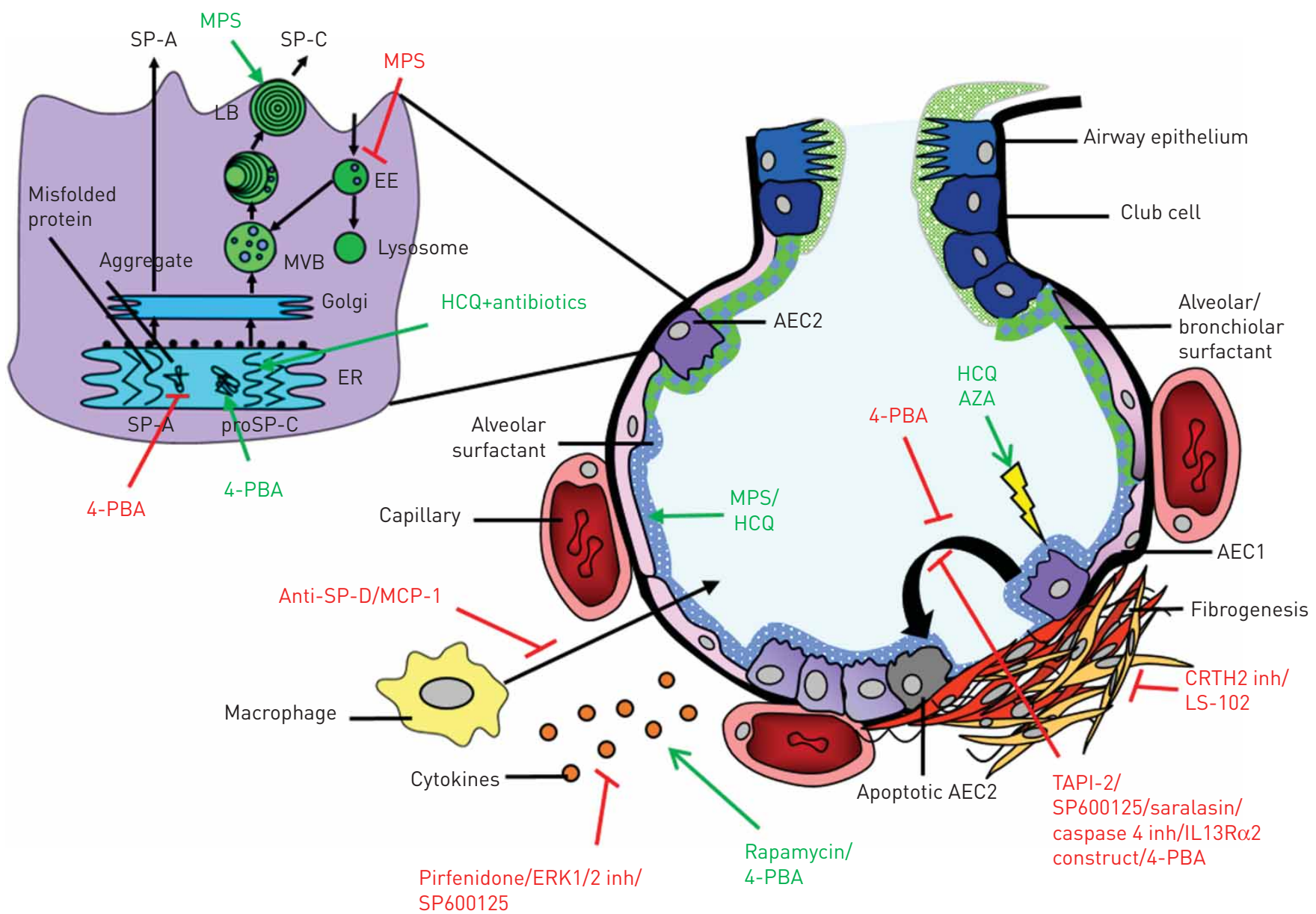

FIGURE 2 Targets of drugs investigated in humans and disease models with a surfactant-processing mutation. Damage to alveolar tissue causes fibrogenesis, which can be targeted by drugs in multiple ways. Expanded section: alveolar type II cell (AEC2) with organelles involved in surfactant processing. MPS: methylprednisolone; SP: surfactant protein; LB: lamellar bodies; EE: early endosomes; MVB: multivesicular bodies; ER: endoplasmic reticulum; 4-PBA: 4-phenylbutyric acid; MCP: monocyte chemotactic protein; ERK: extracellular signal-regulated kinases; HCQ: hydroxychloroquine; AZA: azathioprine; AEC1: alveolar type I cell; inh: inhibitor.

patients with an HPS1 mutation and Hps1ep/Hps1ep, Ap3b1pe/Ap3b1pe (EPPE) C57BL/6J mice treated with anti-MCP1 and/or anti-SP-D. This resulted in reduced macrophage migration [101]. In addition, bleomycin-treated HPS1 mutated mouse AEC2 were treated with CHI3L1, IL13R $\alpha 2$ and CRTH2, which resulted in no effect on apoptosis, amelioration of apoptosis and reduced collagen accumulation, respectively [100].

Outcome in cell lines with a pulmonary surfactant associated mutation

The most frequently studied drugs in cell lines with a surfactant-processing mutation are 4-phenylbutyric acid $(n=5)$, JNK inhibitor $(n=3)$, hydroxychloroquine $(n=3)$, methylprednisolone $(n=2)$, azathioprine $(n=2)$ and cyclophosphamide $(n=2)$. Hydroxychloroquine or methylprednisolone treatment was found to have a positive effect on lysophosphatidylcholine and phosphatidylcholine levels in MLE12 cells transfected with $S_{F T P C}{ }^{\mathrm{I73T}}$ [21] or SFTPC ${ }^{\mathrm{A} 116 \mathrm{D}}$ [57]. In addition, treatment with methylprednisolone, but not hydroxychloroquine, resulted in partial correction of the mislocalisation of pro-SP-C in MLE12 cells expressing SP-C ${ }^{\mathrm{I} 33 \mathrm{~T}}$ [21]. Furthermore, another study with MLE12 cells expressing SP-C $\mathrm{C}^{\mathrm{L} 188 \mathrm{Q}}$ treatment with hydroxychloroquine resulted in increased accumulation of pro-SP-C [56]. Azathioprine seems to have a negative effect on MLE12 cells transfected with $S F T P C^{\mathrm{I} 73 \mathrm{~T}}$ or $S F T P C^{\mathrm{A} 116 \mathrm{D}}$, as evidenced by increased LDH levels after treatment. For cyclophosphamide treatment only an effect on chaperone protein expression of heat shock protein (HSP)70 and HSP90 could be found [21, 57].

In A549 cells transfected with $S F T P C^{\triangle e x o n 4}$, it was found that 4-PBA attenuated increased NF- $\mathrm{KB}$ expression, which is a marker for cellular stress response. However, it had no inhibitory effect on pro-inflammatory interleukin (IL)-8 production [59]. In addition, 4-PBA resulted in reduced NP-40 insoluble aggregate formation and increased protein secretion of $\mathrm{SP}-\mathrm{A} 2^{\mathrm{G} 231 \mathrm{~V}}$ and $\mathrm{SP}-\mathrm{A} 2^{\mathrm{F} 198 \mathrm{~S}}$ expressing 


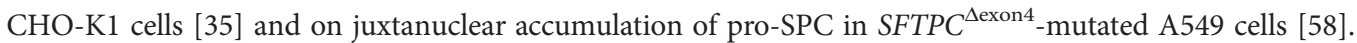
In contrast, 4-PBA resulted in a slightly increased accumulation of SP-C $\mathrm{C}^{\Delta \text { exon4 }}$ and SP-C $\mathrm{C}^{\mathrm{L} 188 \mathrm{Q}}$ proprotein in transfected HEK293 cells. Furthermore, treatment of HEK293 cells transfected with SFTPC ${ }^{\mathrm{L} 188 \mathrm{Q}}$ with 4 -PBA increased endoplasmic reticulum stress and accumulation of detergent insoluble aggregates, but it also resulted in increased mature SP-C $\mathrm{C}^{\mathrm{73} T}$ and SP-C $\mathrm{C}^{\mathrm{L} 188 \mathrm{Q}}$ protein [56]. In contrast, NGUYEN and UHAL [63] showed that treatment of A549 cells transfected with SFTPC ${ }^{\mathrm{G} 100 \mathrm{~S}}$ with 4-PBA resulted in attenuation of increased endoplasmic reticulum stress-induced factors. This study also showed that treatment with 4-PBA can result in reduced nuclear fragmentation.

Another frequently studied drug in cell models is the JNK inhibitor SP600125, sometimes in combination with 4-PBA or a caspase-4 inhibitor. In A549 cells transfected with SFTPC ${ }^{\Delta \text { exon4}}$, this drug resulted in reduced IL-8 concentration [59] and DNA fragmentation [61], although it had no effect on IL-8 concentration in A549 cells transfected with $A B C A 3^{\mathrm{T} 1173 \mathrm{R}}$ [86]. Other drugs not currently used in ILD, e.g. synoviolin inhibitor and saralasin were tested in cell lines with a surfactant-processing mutation by assessing collagen secretion and endoplasmic reticulum-stress induced processes such as apoptosis or expression of chemokines and cytokines. These drugs were only tested in one study, and yielded both positive and negative results.

\section{Discussion}

This systematic review provides an overview of studies that investigated the effect of drugs on patients with ILD and a surfactant-processing mutation or cell or mouse models with a surfactant-processing mutation involved in pulmonary fibrosis. Human studies reported only treatment with antibiotics and drugs against inflammatory or fibrotic processes and evaluated lung function and radiological characteristics over time. Although some positive results were reported in adult case reports/series, except for one case report [10] no curative or long-term stabilising effects on pulmonary fibrosis were reported. In more than half of the case reports/series of children, stabilisation or improvement of disease after treatment was reported. Cell and mouse studies used drugs that interfered with aberrant biological processes related to surfactant mutations. This heterogeneous group of studies showed that results are gene- and mutation-dependent and yielded results that may contribute to the development of personalised medicine in the future.

In two cell line studies with SFTPC $C^{\mathrm{I} 73 \mathrm{~T}}$ - and $S F T P C^{\mathrm{A} 116 \mathrm{D}}$-mutated cells, addition of methylprednisolone showed the most promising results as it partially corrected mislocalisation of SFTPC and (partially) corrected altered (lyso)phospholipid levels [21, 57]. In addition, glucocorticosteroids have been used for the treatment of patients; in three adult cases [7, 10, 89] with a surfactant-processing mutation, improvement or minimal progression of disease was observed after treatment with prednisone. Two of these patients had diseases that are known to often respond to immunosuppressive therapy. One had concomitant pulmonary sarcoidosis [89] and one had hypersensitivity pneumonitis [7]. Two other adult cases $[90,91]$ treated with corticosteroids and pirfenidone showed (short-term) stabilisation of the disease. In paediatric cases described in case reports/series included in this review, treatment with antibiotics, hydroxychloroquine or corticosteroids resulted more often in (short-term) improvement or (short-term) stabilisation of disease compared to adult cases. The difference in response may be due to the difference in clinical phenotype between adult and paediatric patients with surfactant-processing mutations. Children commonly present with desquamative interstitial pneumonia, non-specific interstitial pneumonia, pulmonary alveolar proteinosis, chronic pneumonitis of infancy or respiratory distress syndrome, but seldom with IPF. In addition, host environment, initial injury and regenerative capacity of tissue [104] may differ between adults and children.

It is difficult to draw conclusions only based on case reports/series, since the clinical parameters reported are limited and the information is often not quantitative. In addition, only interesting cases are selected for a case report, introducing bias into the results. Griese and colleagues have initiated a trial to investigate the effect of hydroxychloroquine in paediatric ILD in a more standardised way (ClinicalTrials.gov identifier NCT02615938). A significant subgroup of the patients is expected to carry surfactant-processing mutations; therefore, the results of this trial will provide evidence for therapeutic intervention in paediatric patients with these mutations. However, based on the difference between paediatric and adult patients carrying similar surfactant-processing mutations, translation of these results to clinical management of adult patients will need to proceed with utmost care. In addition, immunosuppressive treatment of adult patients with FIP was shown to be unfavourable in patients with IPF. A negative effect on survival and hospitalisation was found in IPF patients receiving the combination treatment of prednisone, azathioprine and $\mathrm{N}$-acetylcysteine in the PANTHER-IPF trial [105] and recently the negative effect of glucocorticoid treatment was reported for a retrospective cohort of suspected IPF patients [106].

Most reports on patients with a surfactant-processing mutation show that disease progresses as monitored by change deterioration of FVC. Clinical trials were only conducted in HPS patients. In two clinical trials 
the drug pirfenidone was tested. One trial described a positive effect of pirfenidone on slowing down lung function decrease [97], whereas the other clinical trial found no statistically significant difference between the placebo and pirfenidone group [98]. The placebo group of the last clinical trial showed a small rate of decline in FVC. The positive result of the first trial was closely comparable to that reported by KING et al. [107] who showed that pirfenidone is effective in slowing down FVC decrease in a cohort of IPF patients (with unknown genetic characteristics) with a baseline FVC $\geqslant 50 \%$ predicted. The results of both trials included in this review do not provide unambiguous evidence on whether pirfenidone would be helpful for FIP patients with HPS mutations.

In model systems drugs were tested that intervened with the aberrant processes directly related to the surfactant-processing mutation, such as surfactant trafficking, cytokine/chemokine expression, necrosis and apoptosis. The most frequently studied drug in the cell and mouse model studies included in this review is 4-PBA. 4-PBA, a hydrophobic chemical chaperone with a role in promoting trafficking of misfolded proteins, has been approved by the United States Food and Drug Administration for treatment of urea cycle disorders. In addition, its therapeutic effects on other pathologies, such as neurological diseases, diabetes type 2 and protein folding diseases are now being investigated (reviewed by KolB et al. [108]). For 4-PBA a positive effect, and, with other mutations, a negative effect on aggregate formation [35, 56], accumulation of SP-C $[56,58]$ and SP-C mature protein expression $[35,56]$ in different SP-A2- and SP-C-mutated cells has been described. In addition, one study has shown that 4-PBA treatment can reduce nuclear fragmentation in SFTPC ${ }^{\mathrm{G} 100 \mathrm{~S}}$ lung cells [63]. Therefore, further studies are needed to provide evidence for a possible role of 4-PBA in the treatment of patients with surfactant-processing mutations. Other agents, inhibitors against synoviolin, CRTH2, JNK and ANGII receptors showed interesting results in cell studies with a surfactant-processing mutation by reducing collagen secretion/accumulation or nuclear fragmentation. However, future studies, such as replication of cell studies and research in mouse models, including monitoring of side-effects, are still needed.

The investigation of drugs for patients with a SFTPC mutation is complicated by the fact that each pro-SP-C mutation seems to result in unique effects on intracellular trafficking of pro-SP-C and the presence of the mature form of SP-C. Even between mutations that are in the same functional domain of the protein (BRICHOS domain) different effects on SP-C processing have been observed [109]. For example, HEK293 cells transfected with SFTPC ${ }^{\mathrm{I73T}}$ showed increased accumulation of pro-SPC compared to wild-type, whereas SFTPC ${ }^{\mathrm{L} 188 \mathrm{Q}}$ and SFTPC ${ }^{\text {exon4 }}$ showed reduced accumulation of pro-SPC [56].

In summary, different drugs have been tested in different cell lines with a surfactant-processing mutation using different outcome measures. Many of these experiments have only been performed once. To investigate the effect of surfactant-processing mutations and the effect of drugs, the development of a new model for IIPs that better represents affected human lungs is highly wanted. In future, lung organoids, in vitro three-dimensional lung cell models, may fill the gap between cell lines and humans. Tracheobronchial organoids [110] have already been generated from human tissue explants or biopsies. Stable distal lung organoids, which would be necessary to model surfactant processing adequately, have only been generated from tissue derived from mice $[111,112]$. Previously generated distal lung organoids from human lung had a low viability [113], no turnover [114] or high transdifferentiation to type I alveolar epithelial cells [115]. Interestingly, in organoids generated from human-induced pluripotent stem (iPS) cells, alveolar structures have been observed [116, 117]. However, it must be remembered that iPS cells retain characteristics of their cell of origin [118], which might influence their drug response.

In conclusion, this review shows promising drugs described in case reports/series, clinical trials and disease models. One of the two trials in patients with HPS show that patients with surfactant-processing mutations might benefit from anti-fibrotic drugs. Cell and mouse models show that interference with mutation-dependent aberrant processes yield positive results. However, the results seem to be highly geneand mutation-specific. Translation of these results into personalised medicine is not possible at present. Hopefully, the development of new disease model systems with appropriate outcome parameters will make it possible to test drugs on human lung cells with a specific introduced or native surfactant-processing mutation [119], leading to improved treatment strategies for patients with FIP.

Conflict of interest: None declared.

\section{References}

1 Travis WD, Costabel U, Hansell DM, et al. An official American Thoracic Society/European Respiratory Society statement: update of the international multidisciplinary classification of the idiopathic interstitial pneumonias. Am J Respir Crit Care Med 2013; 188: 733-748.

2 García-Sancho C, Buendía-Roldán I, Fernández-Plata MR, et al. Familial pulmonary fibrosis is the strongest risk factor for idiopathic pulmonary fibrosis. Respir Med 2011; 105: 1902-1907. 
Loyd JE. Pulmonary fibrosis in families. Am J Respir Cell Mol Biol 2003; 29: Suppl., S47-S50. van Moorsel CHM, van Oosterhout MFM, Barlo NP, et al. Surfactant protein C mutations are the basis of a significant portion of adult familial pulmonary fibrosis in a Dutch cohort. Am J Respir Crit Care Med 2010; 182: $1419-1425$.

Nogee LM, Dunbar AE, Wert SE, et al. A mutation in the surfactant protein C gene associated with familial interstitial lung disease. N Engl J Med 2001; 344: 573-579.

Nathan N, Giraud V, Picard C, et al. Germline SFTPA1 mutation in familial idiopathic interstitial pneumonia and lung cancer. Hum Mol Genet 2016; 25: 1457-1467.

Wang Y, Kuan PJ, Xing C, et al. Genetic defects in surfactant protein A2 are associated with pulmonary fibrosis and lung cancer. Am J Hum Genet 2009; 84: 52-59.

van Moorsel $\mathrm{CH}$, Ten Klooster $\mathrm{L}$, van Oosterhout $\mathrm{MF}$, et al. SFTPA2 mutations in familial and sporadic idiopathic interstitial pneumonia. Am J Respir Crit Care Med 2015; 192: 1249-1252.

Epaud R, Delestrain C, Louha M, et al. Combined pulmonary fibrosis and emphysema syndrome associated with ABCA3 mutations. Eur Respir J 2014; 43: 638-641.

Campo I, Zorzetto M, Mariani F, et al. A large kindred of pulmonary fibrosis associated with a novel ABCA3 gene variant. Respir Res 2014; 15: 43.

Anderson PD, Huizing M, Claassen DA, et al. Hermansky-Pudlak syndrome type 4 (HPS-4): clinical and molecular characteristics. Hum Genet 2003; 113: 10-17.

Huizing M, Helip-Wooley A, Westbroek W, et al. Disorders of lysosome-related organelle biogenesis: clinical and molecular genetics. Annu Rev Genomics Hum Genet 2008; 9: 359-386.

Gochuico BR, Huizing M, Golas GA, et al. Interstitial lung disease and pulmonary fibrosis in Hermansky-Pudlak syndrome type 2, an adaptor protein-3 complex disease. Mol Med 2012; 18: 56-64.

Weaver TE, Conkright JJ. Function of surfactant proteins B and C. Annu Rev Physiol 2001; 63: 555-578.

66-78.

Mulugeta S, Gray JM, Notarfrancesco KL, et al. Identification of LBM180, a lamellar body limiting membrane protein of alveolar type II cells, as the ABC transporter protein ABCA3. J Biol Chem 2002; 277: 22147-22155.

Carmona-Rivera C, Simeonov DR, Cardillo ND, et al. A divalent interaction between HPS1 and HPS4 is required for the formation of the biogenesis of lysosome-related organelle complex-3 (BLOC-3). Biochim Biophys Acta 2013; 1833: 468-478.

Wei AH, Li W. Hermansky-Pudlak syndrome: pigmentary and non-pigmentary defects and their pathogenesis. Pigment Cell Melanoma Res 2013; 26: 176-192.

Wert SE, Whitsett JA, Nogee LM. Genetic disorders of surfactant dysfunction. Pediatr Dev Pathol 2009; 12: 253-274.

Hawkins A, Guttentag SH, Deterding R, et al. A non-BRICHOS SFTPC mutant (SP-CI73T) linked to interstitia lung disease promotes a late block in macroautophagy disrupting cellular proteostasis and mitophagy. Am J Physiol Lung Cell Mol Physiol 2015; 308: L33-L47.

Woischnik M, Sparr C, Kern S, et al. A non-BRICHOS surfactant protein C mutation disrupts epithelial cell function and intercellular signaling. BMC Cell Biol 2010; 11: 88.

Maitra M, Wang Y, Gerard RD, et al. Surfactant protein A2 mutations associated with pulmonary fibrosis lead to protein instability and endoplasmic reticulum stress. J Biol Chem 2010; 285: 22103-22113.

Lawson WE, Crossno PF, Polosukhin VV, et al. Endoplasmic reticulum stress in alveolar epithelial cells is prominent in IPF: association with altered surfactant protein processing and herpesvirus infection. Am J Physiol Lung Cell Mol Physiol 2008; 294: L1119-L1126.

Tanjore H, Cheng DS, Degryse AL, et al. Alveolar epithelial cells undergo epithelial-to-mesenchymal transition in response to endoplasmic reticulum stress. J Biol Chem 2011; 286: 30972-30980.

Mulugeta S, Maguire JA, Newitt JL, et al. Misfolded BRICHOS SP-C mutant proteins induce apoptosis via caspase-4- and cytochrome C-related mechanisms. Am J Physiol Lung Cell Mol Physiol 2007; 293: L720-L729.

Uhal BD, Nguyen H. The Witschi hypothesis revisited after 35 years: genetic proof from SP-C BRICHOS domain mutations. Am J Physiol Lung Cell Mol Physiol 2013; 305: L906-L911.

Matsumura Y, Ban N, Inagaki N. Aberrant catalytic cycle and impaired lipid transport into intracellular vesicles in ABCA3 mutants associated with nonfatal pediatric interstitial lung disease. Am J Physiol Lung Cell Mol Physiol 2008; 295: L698-L707.

Weichert N, Kaltenborn E, Hector A, et al. Some ABCA3 mutations elevate ER stress and initiate apoptosis of lung epithelial cells. Respir Res 2011; 12: 4.

Dell'Angelica EC, Shotelersuk V, Aguilar RC, et al. Altered trafficking of lysosomal proteins in Hermansky-Pudlak syndrome due to mutations in the $\beta 3$ A subunit of the AP-3 adaptor. Mol Cell 1999; 3: 11-21. Noth I, Zhang Y, Ma SF, et al. Genetic variants associated with idiopathic pulmonary fibrosis susceptibility and mortality: a genome-wide association study. Lancet Respir Med 2013; 1: 309-317.

Fingerlin TE, Murphy E, Zhang W, et al. Genome-wide association study identifies multiple susceptibility loci for pulmonary fibrosis. Nat Genet 2013; 45: 613-620.

Peljto AL, Zhang Y, Fingerlin TE, et al. Association between the MUC5B promoter polymorphism and survival in patients with idiopathic pulmonary fibrosis. JAMA 2013; 309: 2232-2239.

Daccord C, Maher TM. Recent advances in understanding idiopathic pulmonary fibrosis. F1000Res 2016; 5: doi: 10.12688/f1000research.8209.1.

Mathai SK, Newton CA, Schwartz DA, et al. Pulmonary fibrosis in the era of stratified medicine. Thorax 2016 71: $1154-1160$

Song Y, Fang G, Shen H, et al. Human surfactant protein A2 gene mutations impair dimmer/trimer assembly leading to deficiency in protein sialylation and secretion. PLoS One 2012; 7: e46559.

Avital A, Hevroni A, Godfrey S, et al. Natural history of five children with surfactant protein C mutations and interstitial lung disease. Pediatr Pulmonol 2014; 49: 1097-1105.

Rosen DM, Waltz DA. Hydroxychloroquine and surfactant protein C deficiency. N Engl J Med 2005; 352: 207-208. 
Abou Taam R, Jaubert F, Emond S, et al. Familial interstitial disease with I73T mutation: a mid- and long-term study. Pediatr Pulmonol 2009; 44: 167-175.

Kröner C, Reu S, Teusch V, et al. Genotype alone does not predict the clinical course of SFTPC deficiency in paediatric patients. Eur Respir J 2015; 46: 197-206.

Thouvenin G, Abou Taam R, Flamein F, et al. Characteristics of disorders associated with genetic mutations of surfactant protein C. Arch Dis Child 2010; 95: 449-454.

Arıkan-Ayylldız Z, Caglayan-Sozmen S, Isık S, et al. Survival of an infant with homozygous surfactant protein C (SFTPC) mutation. Pediatr Pulmonol 2014; 49: E112-E115.

Hevroni A, Goldman A, Springer C. Infant pulmonary function testing in chronic pneumonitis of infancy due to surfactant protein C mutation. Pediatr Pulmonol 2015; 50: E17-E23.

Sismanlar T, Aslan AT, Griese M. Life-threatening, giant pneumatoceles in the course of surfactant protein C deficiency. Pediatr Pulmonol 2015; 50: E25-E28.

Salerno T, Peca D, Menchini L, et al. Surfactant protein C-associated interstitial lung disease; three different phenotypes of the same SFTPC mutation. Ital J Pediatr 2016; 42: 23.

Brasch F, Griese M, Tredano M, et al. Interstitial lung disease in a baby with a de novo mutation in the SFTPC gene. Eur Respir J 2004; 24: 30-39.

Peca D, Boldrini R, Johannson J, et al. Clinical and ultrastructural spectrum of diffuse lung disease associated with surfactant protein C mutations. Eur J Hum Genet 2015; 23: 1033-1041.

Hime NJ, Fitzgerald D, Robinson P, et al. Childhood interstitial lung disease due to surfactant protein C deficiency: frequent use and costs of hospital services for a single case in Australia. Orphanet J Rare Dis 2014; 9: 36.

Tredano M, Griese M, Brasch F, et al. Mutation of SFTPC in infantile pulmonary alveolar proteinosis with or without fibrosing lung disease. Am J Med Genet A 2004; 126A: 18-26.

Liptzin DR, Patel T, Deterding RR. Chronic ventilation in infants with surfactant protein C mutations: an alternative to lung transplantation. Am J Respir Crit Care Med 2015; 191: 1338-1340.

Mechri M, Epaud R, Emond S, et al. Surfactant protein C gene (SFTPC) mutation-associated lung disease: high-resolution computed tomography (HRCT) findings and its relation to histological analysis. Pediatr Pulmonol 2010; 45: 1021-1029.

Henderson LB, Melton K, Wert S, et al. Large ABCA3 and SFTPC deletions resulting in lung disease. Annals Am Thorac Soc 2013; 10: 602-607.

Banac S, Dessardo S, Lah-Tomulić K, et al. Lethal respiratory failure due to a surfactant protein C gene mutation. Signa Vitae 2012; 7: 47-49.

Chibbar R, Shih F, Baga M, et al. Nonspecific interstitial pneumonia and usual interstitial pneumonia with mutation in surfactant protein C in familial pulmonary fibrosis. Mod Pathol 2004; 17: 973-980.

Percopo S, Cameron HS, Nogee LM, et al. Variable phenotype associated with SP-C gene mutations: fatal case with the I73T mutation. Eur Respir J 2004; 24: 1072-1073.

familial SFTPC mutation. Thorax 2011; 66: 918-919.

Stewart GA, Ridsdale R, Martin EP, et al. 4-Phenylbutyric acid treatment rescues trafficking and processing of a mutant surfactant protein-C. Am J Respir Cell Mol Biol 2012; 47: 324-331.

Zarbock R, Woischnik M, Sparr C, et al. The surfactant protein C mutation A116D alters cellular processing, stress tolerance, surfactant lipid composition, and immune cell activation. BMC Pulm Med 2012; 12: 15.

Wang WJ, Mulugeta S, Russo SJ, et al. Deletion of exon 4 from human surfactant protein C results in aggresome formation and generation of a dominant 36negative. J Cell Sci 2003; 116: 683-692.

Maguire JA, Mulugeta S, Beers MF. Endoplasmic reticulum stress induced by surfactant protein C BRICHOS mutants promotes proinflammatory signaling by epithelial cells. Am J Resp Cell Mol Biol 2011; 44: 404-414.

Madala SK, Maxfield MD, Davidson CR, et al. Rapamycin regulates bleomycin-induced lung damage in SP-C-deficient mice. Pulm Med 2011; 2011: 653534.

Maguire JA, Mulugeta S, Beers MF. Multiple ways to die: delineation of the unfolded protein response and apoptosis induced by surfactant protein C BRICHOS mutants. Int J Biochem Cell Biol 2012; 44: 101-112.

Uhal BD, Nguyen H, Dang M, et al. Abrogation of ER stress-induced apoptosis of alveolar epithelial cells by angiotensin 1-7. Am J Physiol Lung Cell Mol Physiol 2013; 305: L33-L41.

Nguyen H, Uhal BD. The unfolded protein response controls ER stress-induced apoptosis of lung epithelial cells through angiotensin generation. Am J Physiol Lung Cell Mol Physiol 2016; 311: L846-L854.

Nakajima F, Aratani S, Fujita H, et al. Synoviolin inhibitor LS-102 reduces endoplasmic reticulum stress-induced collagen secretion in an in vitro model of stress-related interstitial pneumonia. Int J Mol Med 2015; 35: 110-116. Wittmann T, Frixel S, Höppner S, et al. Increased risk of interstitial lung disease in children with a single R288K variant of ABCA3. Mol Med 2016; 22: 183-191.

Malý J, Navrátilová M, Hornychová $\mathrm{H}$, et al. Respiratory failure in a term newborn due to compound heterozygous ABCA3 mutation: the case report of another lethal variant. J Perinatol 2014; 34: 951-953.

Winter J, Essmann S, Kidszun A, et al. Neonatal respiratory insufficiency caused by an (homozygous) ABCA3-stop mutation: a systematic evaluation of therapeutic options. Klin Padiatr 2014; 226: 53-58.

Doan ML, Guillerman RP, Dishop MK, et al. Clinical, radiological and pathological features of ABCA3 mutations in children. Thorax 2008; 63: 366-373.

Thavagnanam S, Cutz E, Manson D, et al. Variable clinical outcome of ABCA3 deficiency in two siblings. Pediatr Pulmonol 2013; 48: 1035-1038.

Hayes D Jr, Lloyd EA, Fitch JA, et al. ABCA3 transporter deficiency. Am J Respir Crit Care Med 2012; 186: 807. Kitazawa $\mathrm{H}$, Moriya $\mathrm{K}$, Niizuma $\mathrm{H}$, et al. Interstitial lung disease in two brothers with novel compound heterozygous ABCA3 mutations. Eur J Pediatr 2013; 172: 953-957.

M, Annilo T, Ilmoja ML. Different course of lung disease in two siblings with novel ABCA3 mutations. Eur J Pediatr 2014; 173: 1553-1556.

Williamson M, Wallis C. Ten-year follow up of hydroxychloroquine treatment for ABCA3 deficiency. Pediatr Pulmonol 2014; 49: 299-301. 
Thouvenin G, Nathan N, Epaud R, et al. Diffuse parenchymal lung disease caused by surfactant deficiency: dramatic improvement by azithromycin. BMJ Case Rep 2013; 2013: doi: 10.1136/bcr-2013-009988.

Tan JK, Murray C, Schultz A. ABCA3 lung disease in an ex 27 week preterm infant responsive to systemic glucocorticosteroids. Pediatr Pulmonol 2016; 51: E1-E3.

Yokota T, Matsumura Y, Ban N, et al. Heterozygous ABCA3 mutation associated with non-fatal evolution of respiratory distress. Eur J Pediatr 2008; 167: 691-693.

Mukhtar GMA, Al Otaibi WH, Al-Mobaireek KFA, et al. Adenosine triphosphate-binding cassette member A3 gene mutation in children from one family from Saudi Arabia. Ann Thorac Med 2016; 11: 227-229.

Piersigilli F, Peca D, Campi F, et al. New ATP-binding cassette A3 mutation causing surfactant metabolism dysfunction pulmonary type 3. Pediatr Int 2015; 57: 970-974.

Gonçalves JP, Pinheiro L, Costa M, et al. Novel ABCA3 mutations as a cause of respiratory distress in a term newborn. Gene 2014; 534: 417-420.

Carrera P, Ferrari M, Presi S, et al. Null ABCA3 in humans: large homozygous ABCA3 deletion, correlation to clinical-pathological findings. Pediatr Pulmonol 2014; 49: E116-E120.

Uchida DA, Wert SE, Nogee LM, et al. Pulmonary nodules in a newborn with ATP-binding cassette transporter A3 (ABCA3) mutations. Pediatrics 2011; 127: e1347-e1351.

Prestridge A, Wooldridge J, Deutsch G, et al. Persistent tachypnea and hypoxia in a 3-month-old term infant. J Pediatr 2006; 149: 702-706.

Moore GP, Lines MA, Geraghty MT, et al. Novel mutation in ABCA3 resulting in fatal congenital surfactant deficiency in two siblings. Am J Respir Crit Care Med 2014; 189: 750-752.

Shanklin DR, Mullins AC, Baldwin HS. Cerebropulmonary dysgenetic syndrome. Exp Mol Pathol 2008; 85: $112-116$.

Kröner C, Wittmann T, Reu S, et al. Lung disease caused by ABCA3 mutations. Thorax 2017; 72: 213-220.

Flamein F, Riffault L, Muselet-Charlier C, et al. Molecular and cellular characteristics of ABCA3 mutations associated with diffuse parenchymal lung diseases in children. Hum Mol Genet 2012; 21: 765-775.

Hines EJ, Walsh M, Armes JE, et al. Interstitial lung disease in infancy: a general approach. J Paediatr Child Health 2016; 52: 370-376.

Griese M, Haug M, Brasch F, et al. Incidence and classification of pediatric diffuse parenchymal lung diseases in Germany. Orphanet J Rare Dis 2009; 4: 26

Gruson L, Berk T. A case of Hermansky-Pudlak syndrome with pulmonary sarcoidosis. J Clin Aesthet Dermatol 2009; 2: 41-44.

Sakata Y, Kawamura K, Ichikado K, et al. Hermansky-Pudlak syndrome type 4 with interstitial pneumonia. Respir Med Case Rep 2013; 9: 38-41.

Furuhashi K, Enomoto N, Fujisawa $\mathrm{T}$, et al. Hermansky-Pudlak syndrome with nonspecific interstitial pneumonia. Intern Med 2014; 53: 449-453.

Takahashi A, Yokoyama T. Hermansky-Pudlak syndrome with special reference to lysosomal dysfunction. A case report and review of the literature. Virchows Arch A Pathol Anat Histopathol 1984; 402: 247-258.

Kanazu M, Arai T, Sugimoto C, et al. An intractable case of Hermansky-Pudlak syndrome. Intern Med 2014; 53: 2629-2634.

Liza AF, Aziah AM. Albinism and lung fibrosis in a young man - the first case of adult Hermansky-Pudlak syndrome reported in Malaysia. Med J Malaysia 2012; 67: 620-621.

Bachli EB, Brack T, Eppler E, et al. Hermansky-Pudlak syndrome type 4 in a patient from Sri Lanka with pulmonary fibrosis. Am J Med Genet A 2004; 127A: 201-207.

Ideno S, Hatori E, Takeda J, et al. Anesthetic management of a patient with Hermansky-Pudlak syndrome undergoing video-assisted bullectomy. J Clin Anesth 2015; 27: 243-246.

Gahl WA, Brantly M, Troendle J, et al. Effect of pirfenidone on the pulmonary fibrosis of Hermansky-Pudlak syndrome. Mol Genet Metab 2002; 76: 234-242.

O'Brien K, Troendle J, Gochuico BR, et al. Pirfenidone for the treatment of Hermansky-Pudlak syndrome pulmonary fibrosis. Mol Genet Metab 2011; 103: 128-134.

Rouhani FN, Brantly ML, Markello TC, et al. Alveolar macrophage dysregulation in Hermansky-Pudlak syndrome type 1. Am J Respir Crit Care Med 2009; 180: 1114-1121.

Zhou Y, He CH, Herzog EL, et al. Chitinase 3-like-1 and its receptors in Hermansky-Pudlak syndrome-associated lung disease. J Clin Invest 2015; 125: 3178-3192.

Atochina-Vasserman EN, Bates SR, Zhang P, et al. Early alveolar epithelial dysfunction promotes lung inflammation in a mouse model of Hermansky-Pudlak syndrome. Am J Respir Crit Care Med 2011; 184: 449-458.

Young LR, Gulleman PM, Bridges JP, et al. The alveolar epithelium determines susceptibility to lung fibrosis in Hermansky-Pudlak syndrome. Am J Respir Crit Care Med 2012; 186: 1014-1024.

Am J Respir Crit Care Med 2011; 183: 1113.

Clement A, Nathan N, Epaud R, et al. Interstitial lung diseases in children. Orphanet J Rare Dis 2010; 5: 22.

Idiopathic Pulmonary Fibrosis Clinical Research Network, Raghu G, Anstrom KJ, et al. Prednisone, azathioprine, and N-acetylcysteine for pulmonary fibrosis. N Engl J Med 2012; 366: 1968-1977.

Wiertz IA, Wuyts WA, van Moorsel CHM, et al. Unfavourable outcome of glucocorticoid treatment in suspected idiopathic pulmonary fibrosis. Respirology 2018; 23: 311-317.

King TE Jr, Bradford WZ, Castro-Bernardini S, et al. A phase 3 trial of pirfenidone in patients with idiopathic pulmonary fibrosis. N Engl J Med 2014; 370: 2083-2092.

Kolb PS, Ayaub EA, Zhou W, et al. The therapeutic effects of 4-phenylbutyric acid in maintaining proteostasis. Int J Biochem Cell Biol 2015; 61: 45-52.

Thurm T, Kaltenborn E, Kern S, et al. SFTPC mutations cause SP-C degradation and aggregate formation without increasing ER stress. Eur J Clin Invest 2013; 43: 791-800.

Hegab AE, Ha VL, Darmawan DO, et al. Isolation and in vitro characterization of basal and submucosal gland duct stem/progenitor cells from human proximal airways. Stem Cells Transl Med 2012; 1: 719-724. 
111 Barkauskas CE, Cronce MJ, Rackley CR, et al. Type 2 alveolar cells are stem cells in adult lung. J Clin Invest 2013; 123: 3025-3036.

112 Lee JH, Bhang DH, Beede A, et al. Lung stem cell differentiation in mice directed by endothelial cells via a BMP4-NFATc1-thrombospondin-1 axis. Cell 2014; 156: 440-455.

113 Mao P, Wu S, Li J, et al. Human alveolar epithelial type II cells in primary culture. Physiol Rep 2015; 3: e12288.

114 Yu W, Fang X, Ewald A, et al. Formation of cysts by alveolar type II cells in three-dimensional culture reveals a novel mechanism for epithelial morphogenesis. Mol Biol Cell 2007; 18: 1693-1700.

115 Bove PF, Dang H, Cheluvaraju C, et al. Breaking the in vitro alveolar type II cell proliferation barrier while retaining ion transport properties. Am J Respir Cell Mol Biol 2014; 50: 767-776.

116 Huang K, Kang X, Wang X, et al. Conversion of bone marrow mesenchymal stem cells into type II alveolar epithelial cells reduces pulmonary fibrosis by decreasing oxidative stress in rats. Mol Med Rep 2015; 11: $1685-1692$.

117 Hannan NRF, Sampaziotis F, Segeritz CP, et al. Generation of distal airway epithelium from multipotent human foregut stem cells. Stem Cells Dev 2015; 24: 1680-1690.

118 Tabar V, Studer L. Pluripotent stem cells in regenerative medicine: challenges and recent progress. Nat Rev Genet 2014; 15: 82-92.

119 Kropski JA, Blackwell TS, Loyd JE. The genetic basis of idiopathic pulmonary fibrosis. Eur Respir J 2015; 45: $1717-1727$. 